\title{
Megaloblastic Erythropoiesis- An Incidental Finding in Idiopathic Thrombocytopenic Purpura
}

\author{
Dr. I. Sreelakshmi ${ }^{1,}$ Dr. Ezhil Arasi ${ }^{* 2,}$ Dr. Shrinivas. B. $\mathrm{S}^{3,}$ Dr. Shankari $\mathrm{M}^{4}$ \\ ${ }^{1}$ (Associate Professor, Upgraded Department of Pathology, Osmania General Hospital, Hyderabad) \\ ${ }_{2}^{2}$ (Professor and HOD, Upgraded Department of Pathology, Osmania General Hospital, Hyderabad) \\ ${ }^{3}$ (Assistant Professor, Upgraded Department of Pathology, Osmania General Hospital, Hyderabad) \\ ${ }^{4}$ ( Post Graduate $1^{\text {st }}$ Year, Upgraded Department of Pathology, Osmania General Hospital, Hyderabad)
}

\begin{abstract}
Idiopathic thrombocytopenic purpura(ITP) presents as a primary form characterized by isolated thrombocytopenia(platelet count $<100 \times 109 / \mathrm{L})$ in the absence of other causes or disorders that may be associated with thrombocytopenia, or a secondary form in which immune thrombocytopenia develops in association with another disorder that is usually immune or infectious. The diagnosis of ITP is usually a diagnosis by exclusion based on a demonstration of peripheral thrombocytopenia, with a history, physical examination, and complete blood count that do not suggest another cause for the thrombocytopenia ITP may affect individuals of all ages, with peaks during childhood and middle aged. Pallor was the most common clinical manifestation of ITP. The pathogenesis of ITP is complex, involving alterations in humoral and cellular immunity. Thrombocytopenia is caused by antibodies that react with glycoproteins expressed on platelets and megakaryocytes (glycoprotein IIb/IIIa, Ib/IX and others), causing shortened survival of circulating platelets and impairing platelet production. Bone marrow examination done in most of the patients. Micro- normoblastic erythropoiesis found to predominate. Megaloblastic erythropoiesis found in 20.3\% cases. Megaloblastic erythropoiesis- A finding which can be seen in vitamin B12 deficiency, Anti intrinsic factor or Anti gastric mural cell antibodies and may be associated with other disorders of autoimmunity, the most usual being autoimmune thyroid disorders, type I diabetes mellitus, hypoparathyroidism, Addison's disease, post-partum hypophyseal necrosis, ulcerative colitis and vitiligo. The aim of this study is to correlate Bone marrow aspiration findings and possibility of autoimmunity with Idiopathic thrombocytopenic purpura (ITP).
\end{abstract}

Keywords: Idiopathic thrombocytopenic purpura, autoimmunity, antiplatelet antibodies, megaloblastic erythropoiesis,

\section{Introduction}

ITP is a syndrome of various disorders that have in common immune mediated thrombocytopenia but differs in pathogenesis, natural history, comorbidities and responsiveness to therapy. It can be suspected if thrombocytopenia fails to respond to cobalamine therapy or is out of proportion to degree of anaemia.

The diagnosis of ITP is usually a diagnosis by exclusion based on a demonstration of peripheral thrombocytopenia, with a history, physical examination, and complete blood count that do not suggest another cause for the thrombocytopenia. The definition of immune thrombocytopenic purpura (ITP) is modified from that proposed by the Practice Guidelines of the American Society of Hematology for ITP. ITP is defined as thrombocytopenia:(a) in the presence of a normal hemoglobin level, white blood cell count and differential, and blood smear and (b) the absence of other causes of thrombocytopenia.

Slight splenomegaly may be found in patients with ITP . During the last several years, immune thrombocytopenia was also reported in patients with Helicobacter pylori, and platelet counts may or may not normalize with treatment directed only at Helicobacter.

Thrombocytopenia in patients with a variety of solid tumors has also been thought to most likely be immune mediated . Thrombocytopenia may accompany Graves disease and Hashimoto thyroiditis, but it is not certain if it is immunologically mediated. Platelet-associated $\operatorname{IgG}$ has been increased when studied, but there may also be enhanced reticuloendothelial phagocytosis. The pathogenesis of ITP is complex, involving alterations in humoral and cellular immunity. Thrombocytopenia is caused by antibodies that react with glycoproteins expressed on platelets and megakaryocytes (glycoprotein IIb/IIIa, Ib/IX and others), causing shortened survival of circulating platelets and impairing platelet production

Immune complexes have been detected in patients with chronic ITP, but their role in immune platelet destruction remains controversial. The concomitant role of the antibody binding to the same antigens on megakaryocytes and the resultant effect of this binding on platelet production are still unknown.

The pathogenesis of ITP involves loss of tolerance to glycoproteins expressed on platelets and megakaryocytes. Epitope spreading may explain the fact that many patients have antibodies against more than one glycoprotein. 
- Anti-platelet glycoprotein antibodies cause thrombocytopenia through two mechanisms: 1) reducing the survival of circulating platelets, and 2) inhibiting the production of new platelets by bone marrow megakaryocytes.

- Cellular immunity contributes significantly to the pathogenesis of ITP. Alterations in T cell subsets and decreased numbers and activity of regulatory $\mathrm{T}$ cells are common.

Autoantibodies are readily found in plasma or platelet elute in patients with active disease but are infrequently found in patients in remission. Platelet-associated autoantibodies have been detected in $75 \%$ of patients .Serum antiplatelet IgG autoantibodies are detected in approximately 50 to $85 \%$ of patients. IgA serum antiplatelet antibodies appear to be as frequent as IgG, and in approximately

$50 \%$ of cases, both Ig subtypes occur in the same patient. IgM antibodies are also appear.

ITP sera have also been demonstrated to bind to glycosphingolipids and cardiolipin.

Etiology: Infections have a clear role in the initiation of many cases of ITP, at least in childhood. Helicobacter pylori has an important role in the etiology or persistence of ITP. It has been hypothesized that individuals may have an inherited predisposition to ITP. An infection or medication may result in the development of ITP in these individuals.

A component of the etiology of ITP may include immune response genes that predispose patients to develop ITP. A common theme of immune dysregulation, not defined in its specifics, is associated

with many of the diseases linked to ITP. The most obvious example is an inability to make antibodies normally. The mechanism of development of ITP may vary, but one study suggested that autoantibodies

may be restricted to only one $\mathrm{Vh}$ gene family. The hypothesis is that an inability to make antibodies normally(i.e., to use the full $\mathrm{Vh}$ gene repertoire) may result in overutilization of this autoantibody-generating $\mathrm{Vh}$ gene family. These etiologies of ITP could be the result of abnormalities of B cells, but they could equally be explained by abnormal $\mathrm{T}$ cell interactions with B cells.

Defects in the classical pathway of complement may operate via failure to rapidly clear immune complexes, resulting in longer persistence of an "enhanced" immunogen (part of an immune complex). Low C4 has been associated with ITP and low levels of all of the components of the classical pathway have been linked to systemic lupus erythematosus (SLE).

The presence of megaloblastic anaemia can usually be suspected from examination of the peripheral blood and, if features are totally typical, bone marrow aspiration is often not done. The ready availability of accurate assays for vitamin B 12 and folic acid has lessened the importance of bone marrow examination. However, if typical peripheral blood features of megaloblastic erythropoiesis are lacking or if atypical features are present, bone marrow aspiration should be performed. Further tests indicated in patients with megaloblastic anaemia are assays of serum vitamin B 12 and red cell folate followed, when appropriate, by tests for autoantibodies and a Schilling test. If pernicious anaemia is suspected, tests for parietal cell and intrinsic factor antibodies are indicated; the former is more sensitive but less specific.

In summary, a number of possible etiologies, postinfectious and immune predispositions, may underlie the apparent considerable heterogeneity among patients with ITP.

\section{Material And Methods}

A retrospective study conducted at Osmania General Hospital over a period of 3 years, starting from May 2012 to April 2015.Age group included was 15-45years.A total of 376 cases were selected and 325 cases were studied. All patients were having Hemoglobin Range of 4-10g\%.Platelet count less than 1 lakh per microlitres. Out of the 325 ITP cases studied, splenomegaly in 15 patients of non leukemic, non neoplastic origin(symptomatic hypersplenic thrombocytopenia).In 295 cases, bone marrow aspiration was done using Salah's needle, site of aspiration being ilial crest or sternum to rule the cause of anaemia. Complete blood count obtained using 3 part automated hematology analyzer, Sysmex. Peripheral blood smear examination was done for presence of anaemia, leucocyte abnormalities. Coomb's test to exclude autoimmune hemolytic anaemia.

Thrombocytopenia secondary to other causes such as pseudo thrombocytopenia due to EDTA, Liver diseases, Acute or chronic leukemia, Myelodysplasia, Aplastic anaemia , Drugs intake, Lack of sufficient data \& irregular follow up were excluded from the study.

\section{Results}

Our study on 325 patients showed the most common age group involved was 15-30years(Figure no 1). Female preponderance was noticed with an average of $75 \%$. (Figure no. 2 In our study, we could find that pallor was the most common clinical manifestation (Table no 1. Peripheral blood examination(Figure no 3) was done in all patients. $20.3 \%$ of the cases showed anisopoikilocytosis,

with predominantly macrocytes, ovalocytes, thrombocytopenia, hypersegmented neutrophils in few. In all cases in whom anaemia was seen, bone marrow aspiration was carried out to rule out other causes. Bone marrow 
examination revealed normoblastic, microblastic, megaloblastic erythropoiesis. Few showing micronormoblastic picture(Figure no4). Microcytic picture was predominant, amounting to $38 \%$. Iron deficiency anaemia could be attributed to it. However, Megaloblastic erythropoiesis was identified in $20.3 \%$ cases. Bone marrow examination in patients with megaloblastic picture showed increased megakaryocytes(figure no.5), immature megakaryocytes with large non lobulated nuclei ( Figure no6 ), megaloblasts (Figure no $7 \& 8$ ), giant metamyelocytes were seen along with cells of erythroid lineage. The bone marrow in megaloblastic anemia is hypercellular, with erythroid precursors predominating. Iron stores were evaluated by Perl's stain, which was either normal or decreased.

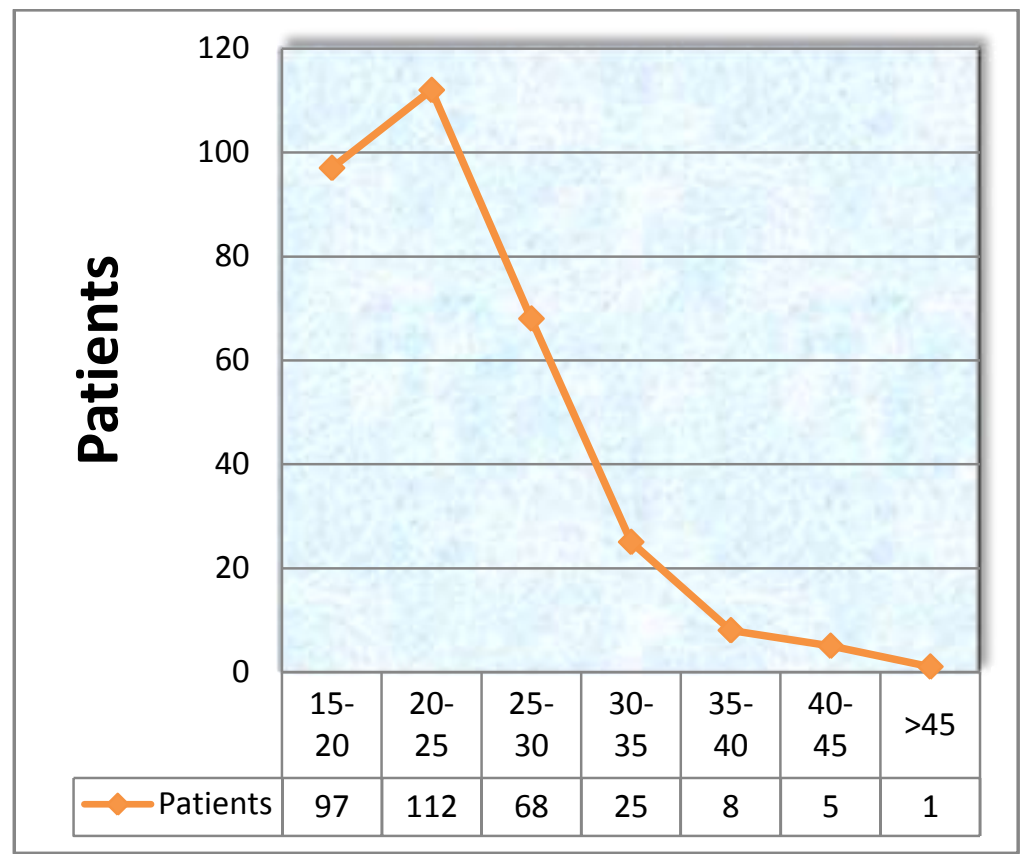

Figure no.1 : Age vs number of patients in all age groups.

\section{Incidence of Cases}

$\square$ Incidence of cases Male $\square$ Incidence of cases Female

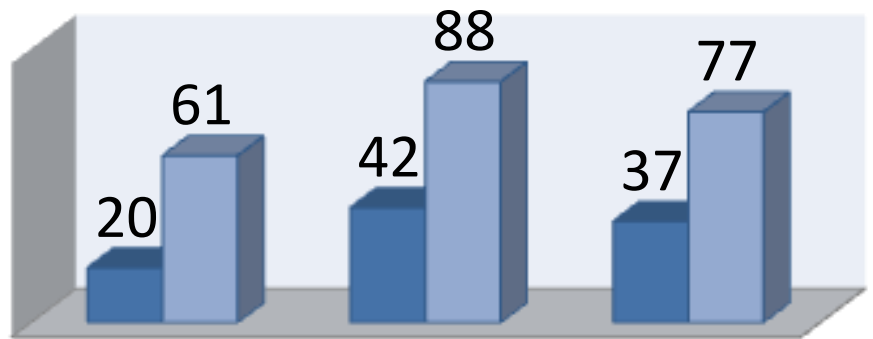

\section{$2012-13 \quad 2013-14 \quad 2014-15$}

Figure no 2: Incidence of ITP in females and males during 3years study. 


\begin{tabular}{|c|c|c|}
\hline \multicolumn{3}{|c|}{ Percentage Incidence of Clinical Feature } \\
\hline $\begin{array}{c}\text { Clinical } \\
\text { Feature }\end{array}$ & $\begin{array}{c}\text { Total number of } \\
\text { Incident cases }\end{array}$ & $\begin{array}{c}\text { \% of } \\
\text { Incidence }\end{array}$ \\
\hline Pallor & 295 & 90 \\
\hline Gum Bleeding & 238 & 73.6 \\
\hline $\begin{array}{c}\text { Petechial } \\
\text { Hemorrhage }\end{array}$ & 178 & 54.7 \\
\hline Epistaxis & 58 & 17.8 \\
\hline Bleeding P/V & 28 & 8.6 \\
\hline Splenomegaly & 15 & 4.6 \\
\hline $\begin{array}{c}\text { Spontaneous } \\
\text { Abortions }\end{array}$ & 12 & 3.6 \\
\hline Hypertension & 6 & 1.6 \\
\hline Malena & 5 & 1.5 \\
\hline Hematuria & 4 & 1.2 \\
\hline Jaundice & 2 & 0.6 \\
\hline
\end{tabular}

Table no 1: Percentage incidence of clinical cases in ITP

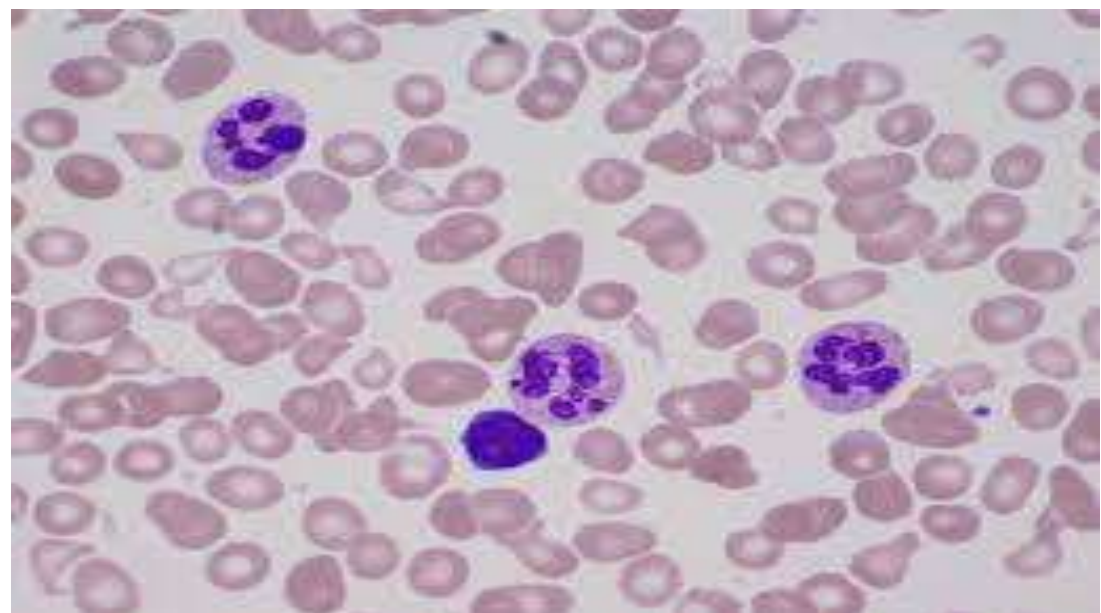

Figure no 3: Peripheral blood smear examination showing Anisocytosis with predominant macrocytes, ovalocytes, hypersegmented neutrophils, thrombocytopenia.

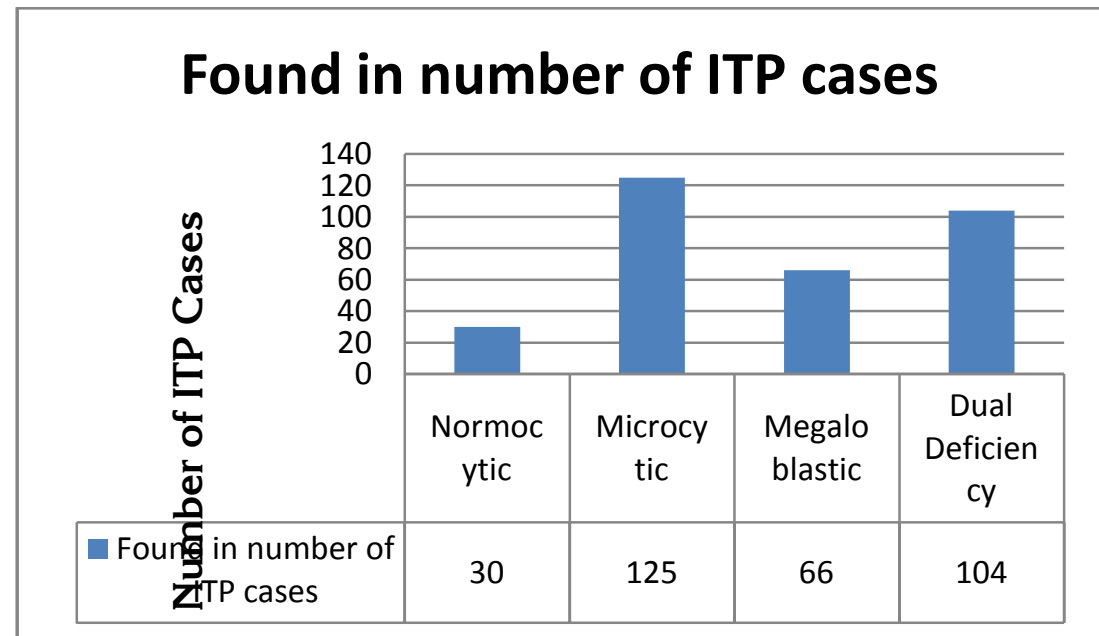

Figure no 4:Incidence of Bone marrow findings in ITP 


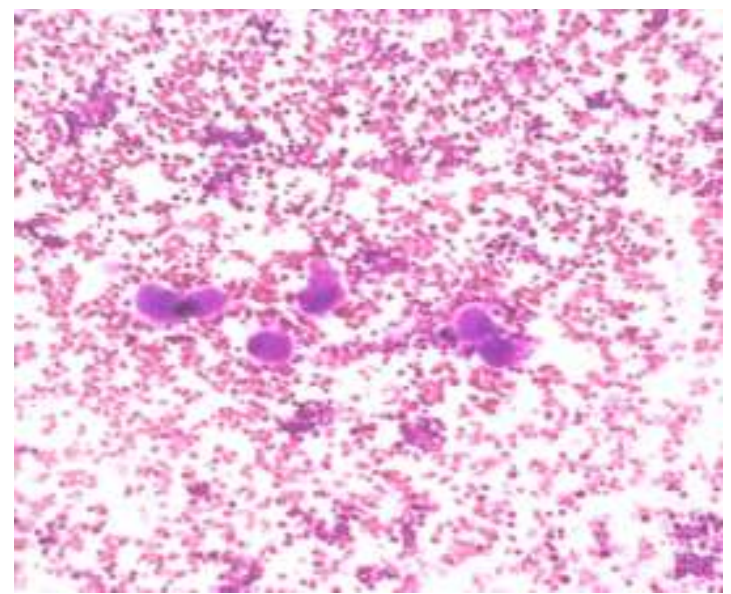

Figure no 5: Bone marrow examination,10x

Magnification showing Incresed megakaryocytes

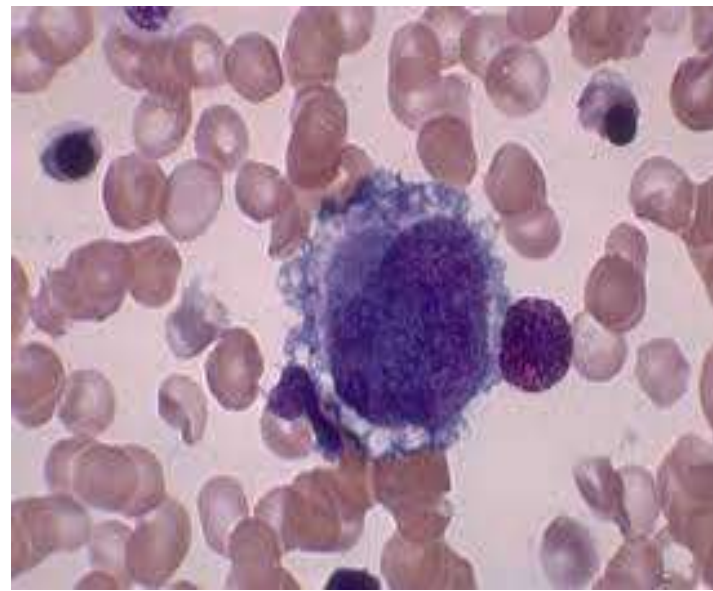

Figure no 6: Bone marrow examination , 100x magnification showing immature megakaryocyte
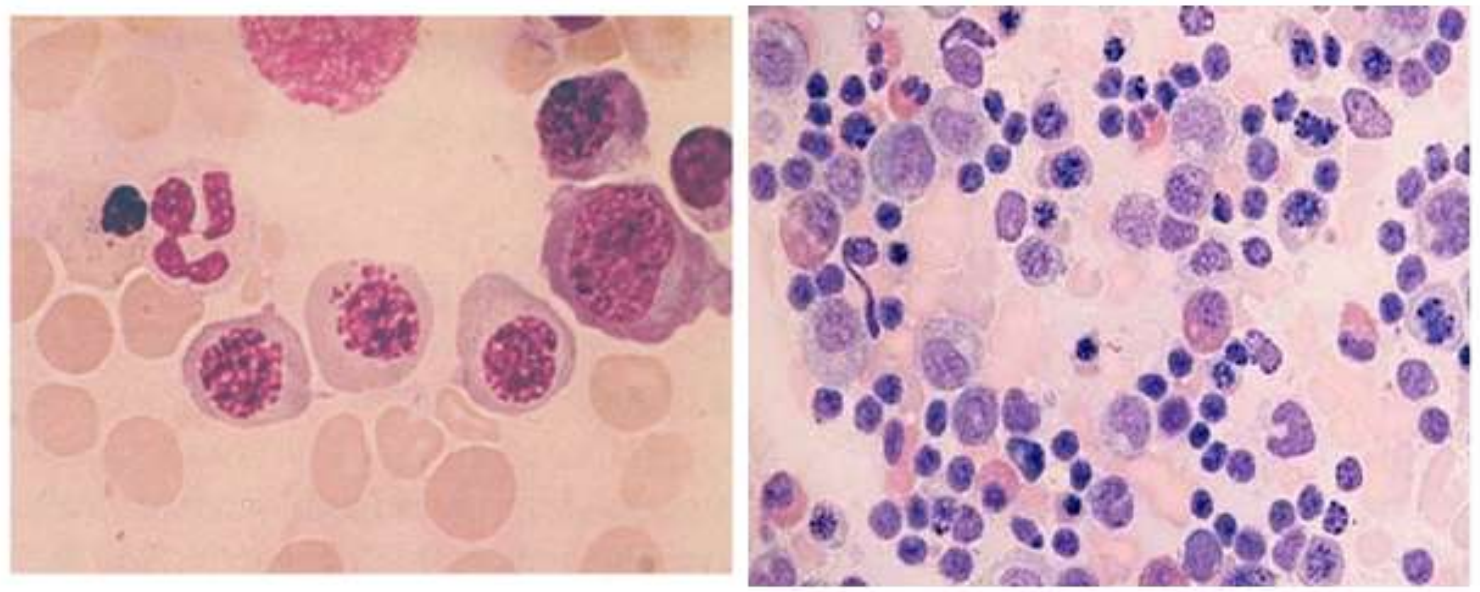

Figure no 7 : Bone marrow examination, 100x

Figure no 8: Bone marrow examination , $40 \mathrm{x}$ Magnification showing megaloblasts and giant metamyelocyte.

\begin{tabular}{|c|c|c|c|}
\hline itrbutas & 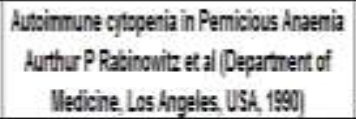 & 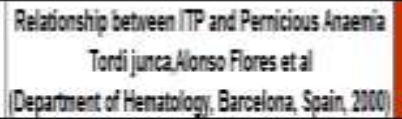 & 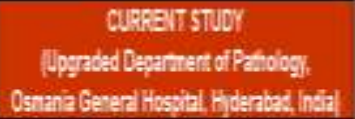 \\
\hline Casesurter stid. & 4 & 231 & 365 \\
\hline Age Grup ("eass) & 2888 & $25-65$ & 185 \\
\hline $\begin{array}{c}\text { Sex huderce Fendes } \\
\text { Wides) }\end{array}$ & 3Fendes, Illate & 120 Fendes, 1911 las & 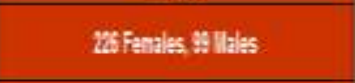 \\
\hline Hencolokin Pave (ghi) & 3595 & की & 4t. \\
\hline Platel Cort & $2000-12$ alt & $4121 \mathrm{ak}$ & 4 bat \\
\hline legalanuctecost & Nomd or huesed & Nomral or horessed & Nomal or heressed \\
\hline $\begin{array}{l}\text { Bunellativu Aspradion } \\
\text { Fndrgs }\end{array}$ & 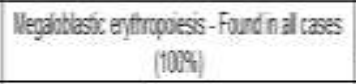 & 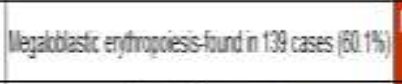 & 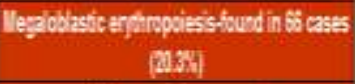 \\
\hline mon Steres & Cexeased & Fiamal o Oerreased & Nama ir Denased \\
\hline Counts Test & liegative & lot perlymed & Meztive \\
\hline Pesertrng Simqtums & 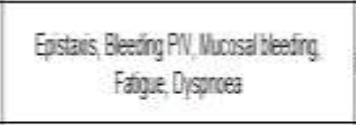 & 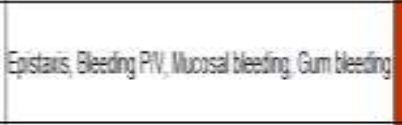 & 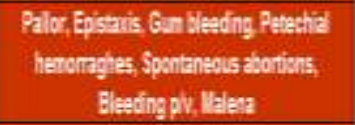 \\
\hline Othe hestigaions & None & 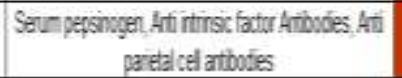 & None: \\
\hline
\end{tabular}

Table no 2 : Comparitive study of our study with Rabinowitz et al and Tordi junca et al study. 


\section{Discussion}

Our study was compared with the study done by Rabinowitz et al (Department of medicine, Los Angeles,USA,1990 ) and Tordi junca et al (Department of hematology, Barcelona, Spain, 2000), which also revealed female preponderance. Other studies have suggested that there is a 2 to $3: 1$ female to male predominance, consistent with the generally recognized increased incidence of autoimmune disease in women and a peak age of onset of ITP in women of child-bearing age.

In Rabinowitz et al and Tordi junca et al study, bleeding gums was most common. Bone marrow examination revealed Microcytic picture was predominant, amounting to $38 \%$. Iron deficiency anaemia could be attributed to it. However, Megaloblastic erythropoiesis was identified in $20.3 \%$ cases. In the study by Rabinowitz et al and Tordi junca et al, Megaloblastic erythropoiesis was seen in $100 \%$ and $60 \%$ cases respectively(Table no 2). Bone marrow examination in patients with megaloblastic picture showed increased megakaryocytes(figure no.5), immature megakaryocytes with large non lobulated nuclei ( Figure no6 ), megaloblasts (Figure no7\&8), giant metamyelocytes were seen along with cells of erythroid lineage. The bone marrow in megaloblastic anemia is hypercellular, with erythroid precursors predominating. Iron stores evaluated by Perl's stain, was either normal or decreased, which coincided with Rabinowitz et al study and Tordi junca et al study.

\section{Conclusion}

There is no diagnostic blood test (e.g., antiplatelet antibodies) that reliably and consistently includes or excludes ITP. An algorithm for the diagnosis of ITP could proceed in three steps. A) Distinguish ITP from systemic disorders causing thrombocytopenia as part of their pathophysiology. B) Distinguish immune thrombocytopenia from nonimmune thrombocytopenia. C) Distinguish primary ITP from secondary ITP i.e, distinguishing primary, "idiopathic" ITP from ITP secondary to other diseases such as SLE, HIV, CLL, and CVID, drug induced thrombocytopenia.

Other tests, in addition to a bone marrow examination, include Reticulocyte count, to help exclude both Thrombotic thrombocytopenic purpura and Evans syndrome (autoimmune anemia in association with ITP). Antinuclear antibody (ANA) and antidouble stranded DNA antibody to investigate the possibility of SLE. Prothrombin time, partial thromboplastin time, fibrinogen, and D-dimer to consider DIC or other nonimmune condition. Radiologic studies (e.g., CT scan) to search for lymphoma or other malignancy.

In summary, ITP is usually an autoantibody-mediated disease that results in often severe thrombocytopenia. ITP has the virtue of having an easily obtainable and highly specific surrogate marker for hemorrhage: the platelet count. Fortunately, even at low platelet counts, serious hemorrhage (e.g., intracranial) is rare. Difficulties in diagnosis include the lack of a specific platelet antibody test. Other tests (e.g., plasma TPO levels, reticulated platelet counts, and the number of large platelets) are being developed to provide complementary pathophysiological information .Autoimmunity in case of megaloblastic picture should be considered. Current management is therefore largely based on an individual patient's degree of bleeding and response to previous treatments. There are a number of standard treatments. Methods to acutely increase the platelet count are numerous and generally effective, including high-dose steroids, IVIG, and higher dose, 75 $\mu \mathrm{g} / \mathrm{kg}$ IV anti-D. In addition, there appears to be a clear benefit of combining agents in difficult patients. Maintenance of the platelet count prior to splenectomy, or when splenectomy (the only curative treatment in more than $50 \%$ of patients) has failed, is the subject of ongoing studies but the upfront treatments and especially the novel thrombopoietic agents seem useful.

\section{References}

[1]. Rabinowitz AP, Sacks Y, Carmel R. Autoimmune cytopenias in pernicious anemia: A report of four cases and review of the literature.Eur J Haematol 1990: 44: 18-23

[2]. Tordi JuncaÁ ,1 Alonso Flores,1 Maria Luisa Granada,2 Orlando JimeÂnez2 and Juan M. Sancho1 1; The relationship between idiopathic thrombocytopenic purpura and pernicious anaemia;, Hospital Universitari Germans Trias i Pujol, Badalona (Barcelona), Spain; British Journal of Haematology, 2000, 111, 513-516

[3]. Kuwana M, Kurata Y, Fujimura K, Fujisawa K, Wada H, NagasaT, Nomura S, Kojima T, Yagi H, Ikeda Y. Preliminary laboratory based diagnostic criteria for immune thrombocytopenic purpura: evaluation by multi-center prospective study. J Thromb Haemost 2006;

[4]. James B. Bussel; Immune thrombocytopenic purpura; Department of pediatrics, Weil medical college of Cornell university, New York Presbyterian hospital, New York.

[5]. William Dameshek, M.D., and Captain Edward B. Miller, A.U.S. The Megarkaryocytes in Idiopathic Thrombocytopenic Purpura, A form of Hypersplenism. www.bloodjournal.org; July 15, 2015 